\title{
Solving Global Optimization Problems over Polynomials with GloptiPoly 2.1
}

\author{
Didier Henrion $^{1,2}$ and Jean-Bernard Lasserre ${ }^{1}$ \\ 1 Laboratoire d'Analyse et d'Architecture des Systèmes, Centre National de la \\ Recherche Scientifique, 7 Avenue du Colonel Roche, 31077 Toulouse, cedex 4, France \\ \{henrion, lasserre\}@laas.fr \\ 2 Institute of Information Theory and Automation, Academy of Sciences of the \\ Czech Republic, Pod vodárenskou věží 4, 18208 Praha, Czech Republic \\ henrion@utia.cas.cz
}

\begin{abstract}
GloptiPoly is a Matlab/SeDuMi add-on to build and solve convex linear matrix inequality relaxations of the (generally non-convex) global optimization problem of minimizing a multivariable polynomial function subject to polynomial inequality, equality or integer constraints. It generates a series of lower bounds monotonically converging to the global optimum. Global optimality is detected and isolated optimal solutions are extracted automatically. In this paper we first briefly describe the theoretical background underlying the relaxations. Following a small illustrative example of the use of GloptiPoly, we then evaluate its performance on benchmark test examples from global optimization, combinatorial optimization and polynomial systems of equations.
\end{abstract}

\section{Introduction}

GloptiPoly is a Matlab ${ }^{3}$ freeware that builds and solves convex linear matrix inequality (LMI, see [VB96]) relaxations of (generally non-convex) global optimization problems with multivariable real-valued polynomial objective function and constraints. The software solves a series of convex relaxations of increasing size, whose optima are guaranteed to converge monotonically to the global optimum of the original non-convex optimization problem.

GloptiPoly solves LMI relaxations with the help of the semidefinite programming (SDP) solver SeDuMi [SDM99], taking full advantage of sparsity and special problem structure. Optionally, a user-friendly interface called DefiPoly, based on Matlab Symbolic Math Toolbox, can be used jointly with GloptiPoly to define the optimization problems symbolically with a Maple-like syntax.

GloptiPoly is aimed at small- and medium-scale problems. Numerical experiments illustrate that for most of the problem instances available in the literature, the global optimum is reached exactly with LMI relaxations of medium size, at a relatively low computational cost.

\footnotetext{
${ }^{3}$ Matlab is a trademark of The MathWorks, Inc.
} 
GloptiPoly requires Matlab version 5.3 or higher [Mat01], together with the freeware solver SeDuMi version 1.05 [SDM99]. For installation instructions and a comprehensive user's guide, see

wWw.laas.fr/ henrion/software/gloptipoly

\section{Theoretical background}

GloptiPoly is based on the theory of positive polynomials and moments described in [Las01,Las02] and briefly summarized in the sequel.

\subsection{Introduction}

Consider the general nonlinear optimization problem

$$
\mathbb{P} \rightarrow p^{*}:=\min _{x \in \mathbb{R}^{n}}\left\{g_{0}(x) \mid g_{k}(x) \geq 0, k=1, \ldots m\right\}
$$

where all the $g_{k}(x): \mathbb{R}^{n} \rightarrow \mathbb{R}$ are real-valued polynomials of $\mathbb{R}\left[x_{1}, \ldots, x_{n}\right]$. Equality constraints are allowed via two opposite inequalities, so that (1) describes all optimization problems that involve polynomials. In particular, it encompasses non-convex quadratic problems as well as discrete optimization problems (e.g. 0-1 nonlinear programming problems).

The idea behind the methodology of GloptiPoly is to build up a sequence of convex semidefinite relaxations of $\mathbb{P}$ of increasing size and whose sequence of optimal values converges to the global optimal value $p^{*}=\inf \mathbb{P}$.

The original idea can be traced back to the pioneering Reformulation Linearization Technique (RLT) of [SA90,SA99] where additional redundant constraints (products of the original ones) are introduced and linearized in a higher space (lifting) by introducing additional variables (e.g. $x_{i} x_{j}=y_{i j}$ ) so as to obtain a LP-relaxation. Convergence was proved for 0-1 nonlinear programs. Later, Shor [Sho87,Sho98] also proposed a lifting procedure to reduce any polynomial programming problem to a quadratic one and then use a semidefinite relaxation to obtain a lower bound of $p^{*}$, see also the more recent work [Nes00]. Then, the striking certified good approximation of Goemans and Williamson for the MAX-CUT problem [GW95], obtained from a simple SDP (or LMI) relaxation definitely excited the curiosity of researchers for SDP relaxations. However, excepted for the LP-relaxations of Sherali and Adams in 0-1 problems, no proof of convergence was provided.

The proof of convergence of the LMI relaxations defined in [Las01,Las02] and used in GloptiPoly is based on recent results of real algebraic geometry concerning the representation of polynomials, strictly positive on a semi-algebraic set; see also [Par00] for a related approach. It turns out that the primal and dual LMI relaxations of GloptiPoly match both sides of the dual theories of moments and positive polynomials.

Indeed, while the primal relaxations aim at founding the moments of a probability measure with mass concentrated on some global minimizers of $\mathbb{P}$, the 
dual relaxations aim at representing the polynomial $g_{0}(x)-p^{*}$, nonnegative on the (semi-algebraic) feasible set $\mathbb{K}$ of $\mathbb{P}$, as a linear combination of the $g_{i}$ 's with weights being polynomials that are sums of squares, as in Putinar's representation of polynomials, strictly positive on a semi-algebraic set [Put93].

In brief, the primal LMI relaxations $\left\{\mathbb{Q}_{i}\right\}$ of $\mathbb{P}$ are relaxations of the problem (equivalent to $\mathbb{P}$ )

$$
p^{*}=\min _{\mu}\left\{\int g_{0} d \mu \mid \mu(\mathbb{K})=1\right\},
$$

where the minimum is taken over all the probability measures on the feasible set $\mathbb{K}$ of $\mathbb{P}$, whereas the dual relaxations $\left\{\mathbb{Q}_{i}^{*}\right\}$ solve

$$
\max _{\rho_{i},\left\{q_{k}\right\}}\left\{\rho_{i} \mid g_{0}(x)-\rho_{i}=q_{0}+\sum_{k=1}^{m} g_{k}(x) q_{k}(x)\right\},
$$

where the unknowns $\left\{q_{k}\right\}$ are polynomials, all sums of squares, and with degree at most $2 i$. For a brief account of these two dual points of view, the interested reader is referred to [Las01,Las02] and the references therein.

The increasing size of the relaxations reflects the effort in the degree $2 i$ needed in (2) for $\rho_{i}$ to be as closed as desired to $p^{*}$ (and often to be exactly equal to $\left.p^{*}\right)$.

\subsection{Brief description of the methodology}

Notation and definitions: Given any two real-valued symmetric matrices $A, B$ let $\langle A, B\rangle$ denote the usual scalar product $\operatorname{trace}(A B)$ and let $A \succeq B$ (resp. $A \succ B$ ) stand for $A-B$ positive semidefinite (resp. $A-B$ positive definite). Let

$$
1, x_{1}, x_{2}, \ldots x_{n}, x_{1}^{2}, x_{1} x_{2}, \ldots, x_{1} x_{n}, x_{2}^{2}, x_{2} x_{3}, \ldots, x_{n}^{2}, \ldots, x_{1}^{r}, \ldots, x_{n}^{r},
$$

be a basis for the space $\mathcal{A}_{r}$ of real-valued polynomials of degree at most $r$, and let $s(r)$ be its dimension. Therefore, a polynomial $p: \mathbb{R}^{n} \rightarrow \mathbb{R}$ of degree $r$ is written

$$
p(x)=\sum_{\alpha} p_{\alpha} x^{\alpha}, \quad x \in \mathbb{R}^{n},
$$

where

$$
x^{\alpha}=x_{1}^{\alpha_{1}} x_{2}^{\alpha_{2}} \ldots x_{n}^{\alpha_{n}}, \quad \text { with } \sum_{i=1}^{n} \alpha_{i}=k,
$$

is a monomial of degree $k$ with coefficient $p_{\alpha}$. Let $p=\left\{p_{\alpha}\right\} \in \mathbb{R}^{s(r)}$ be the vector of coefficients of the polynomial $p(x)$ in the basis $(3)$.

Given an $s(2 r)$-sequence $\left(1, y_{1}, \ldots,\right)$, let $M_{r}(y)$ be the moment matrix of dimension $s(r)$ with rows and columns indexed by (3). For instance, to fix ideas, consider the 2-dimensional case. The moment matrix $M_{r}(y)$ is the block matrix $\left\{M_{i, j}(y)\right\}_{0 \leq i, j \leq r}$ defined by

$$
M_{i, j}(y)=\left[\begin{array}{cccc}
y_{i+j, 0} & y_{i+j-1,1} & \ldots & y_{i, j} \\
y_{i+j-1,1} & y_{i+j-2,2} & \ldots & y_{i-1, j+1} \\
\ldots & \ldots & \ldots & \ldots \\
y_{j, i} & y_{i+j-1,1} & \ldots & y_{0, i+j}
\end{array}\right] .
$$


Thus, with $n=2$ and $r=2$, one obtains

$$
M_{2}(y)=\left[\begin{array}{l|ll|lll}
1 & y_{10} & y_{01} & y_{20} & y_{11} & y_{0,2} \\
\hline y_{10} & y_{20} & y_{11} & y_{30} & y_{21} & y_{12} \\
y_{01} & y_{11} & y_{02} & y_{21} & y_{12} & y_{03} \\
\hline y_{20} & y_{30} & y_{21} & y_{40} & y_{31} & y_{22} \\
y_{11} & y_{21} & y_{12} & y_{31} & y_{22} & y_{13} \\
y_{02} & y_{12} & y_{03} & y_{22} & y_{13} & y_{04}
\end{array}\right]
$$

Another, more intuitive way of constructing $M_{r}(y)$ is as follows. If $M_{r}(y)(1, i)=$ $y_{\alpha}$ and $M_{r}(y)(j, 1)=y_{\beta}$, then $M_{r}(y)(i, j)=y_{\alpha+\beta}$, with $\alpha+\beta=\left(\alpha_{1}+\right.$ $\left.\beta_{1}, \cdots, \alpha_{n}+\beta_{n}\right)$. This defines a bilinear form $\langle., .\rangle_{y}$ on $\mathcal{A}_{r}$, by $\langle q(x), v(x)\rangle_{y}:=$ $\left\langle q, M_{r}(y) v\right\rangle, \quad q(x), v(x) \in \mathcal{A}_{r}$, and if $y$ is a sequence of moments of some measure $\mu_{y}$, then

$$
\left\langle q, M_{r}(y) q\right\rangle=\int q(x)^{2} \mu_{y}(d x) \geq 0,
$$

so that $M_{r}(y) \succeq 0$.

If the entry $(i, j)$ of the matrix $M_{r}(y)$ is $y_{\beta}$, let $\beta(i, j)$ denote the subscript $\beta$ of $y_{\beta}$. Next, given a polynomial $\theta(x): \mathbb{R}^{n} \rightarrow \mathbb{R}$ with coefficient vector $\theta$, we define the matrix $M_{r}(\theta y)$ by

$$
M_{r}(\theta y)(i, j)=\sum_{\alpha} \theta_{\alpha} y_{\{\beta(i, j)+\alpha\}} .
$$

For instance, with

$$
M_{1}(y)=\left[\begin{array}{c|cc}
1 & y_{10} & y_{01} \\
\hline y_{10} & y_{20} & y_{11} \\
y_{01} & y_{11} & y_{02}
\end{array}\right] \text { and } x \mapsto \theta(x):=a-x_{1}^{2}-x_{2}^{2},
$$

we obtain

$$
M_{1}(\theta y)=\left[\begin{array}{c|c|c}
a-y_{20}-y_{02} & a y_{10}-y_{30}-y_{12} a y_{01}-y_{21}-y_{03} \\
\hline a y_{10}-y_{30}-y_{12} & a y_{20}-y_{40}-y_{22} a y_{11}-y_{31}-y_{13} \\
a y_{01}-y_{21}-y_{03} & a y_{11}-y_{31}-y_{13} a y_{02}-y_{22}-y_{04}
\end{array}\right] .
$$

In a manner similar to what we have in (4), if $y$ is a sequence of moments of some measure $\mu_{y}$, then

$$
\left\langle q, M_{r}(\theta y) q\right\rangle=\int \theta(x) q(x)^{2} \mu_{y}(d x),
$$

for every polynomial $q(x): \mathbb{R}^{n} \rightarrow \mathbb{R}$ with coefficient vector $q \in \mathbb{R}^{s(r)}$. Therefore, $M_{r}(\theta y) \succeq 0$ whenever $\mu_{y}$ has its support contained in the set $\{\theta(x) \geq 0\}$. The matrix $M_{r}(\theta y)$ is called a localizing matrix.

The $\mathbb{K}$-moment problem identifies those sequences $y$ that are moment-sequences of a measure with support contained in the semi-algebraic set $\mathbb{K}$. In duality with the theory of moments is the theory of representation of positive polynomials, which dates back to Hilbert's 17th problem. This fact will be reflected in the semidefinite relaxations proposed later. 
LMI relaxations: Let $\mathbb{P}$ be the problem defined in (1) and let

$$
\mathbb{K}:=\left\{x \in \mathbb{R}^{n} \mid g_{k}(x) \geq 0, k=1, \ldots, m\right\}
$$

be the feasible set associated with $\mathbb{P}$.

Depending on its parity, let degree $\left(g_{k}\right)=2 v_{k}-1$ or $2 v_{k}$, for all $k=0,1, \ldots, m$. When needed below, for $i \geq \max _{k} v_{k}$, the vectors $g_{k} \in \mathbb{R}^{s\left(2 v_{k}\right)}$ are extended to vectors of $\mathbb{R}^{s(2 i)}$ by completing with zeros. As we minimize $g_{0}(x)$ we may and will assume that its constant term is zero, that is, $g_{0}(0)=0$.

For $i \geq \max _{k \in\{0, m\}} v_{k}$, consider the following family $\left\{\mathbb{Q}_{i}\right\}$ of convex positive semidefinite programs, or LMI relaxations of $\mathbb{P}$ :

$$
\mathbb{Q}_{i}\left\{\begin{aligned}
\min _{y} \sum_{\alpha}\left(g_{0}\right)_{\alpha} y_{\alpha} & \\
M_{i}(y) & \succeq 0 \\
M_{i-v_{k}}\left(g_{k} y\right) & =0, \quad k=1, \ldots, m,
\end{aligned}\right.
$$

with respective dual problems

$$
\mathbb{Q}_{i}^{*}\left\{\begin{array}{r}
\min _{X \succeq 0, Z_{k}}-X(1,1)-\sum_{k=1}^{m} g_{k}(0) Z_{k}(1,1) \\
\left\langle X, B_{\alpha}\right\rangle+\sum_{k=1}^{m}\left\langle Z_{k}, C_{\alpha}^{k}\right\rangle=\left(g_{0}\right)_{\alpha}, \forall \alpha \neq 0
\end{array}\right.
$$

where $X, Z_{k}$ are real-valued symmetric matrices, the "dual variables" associated with the constraints $M_{i}(y) \succeq 0$ and $M_{i-v_{k}}\left(g_{k} y\right) \succeq 0$ respectively, and where we have written

$$
M_{i}(y)=\sum_{\alpha} B_{\alpha} y_{\alpha} ; M_{i-v_{k}}\left(g_{k} y\right)=\sum_{\alpha} C_{\alpha}^{k} y_{\alpha}, k=1, \ldots, n,
$$

for appropriate real-valued symmetric matrices $B_{\alpha}, C_{\alpha}^{k}, k=1, \ldots, n$.

In the standard terminology, the constraint $M_{i}(y) \succeq 0$ is called a linear matrix inequality (LMI) and $\mathbb{Q}_{i}$ and its dual $\mathbb{Q}_{i}^{*}$ are so-called positive semidefinite programs, the LMI relaxations of $\mathbb{P}$. The reader interested in more details on SDP and LMIs is referred to [VB96] and the many references therein.

Remark: In the case of 0-1 programming (and more generally, discrete optimization problems) the relaxations $\mathbb{Q}_{i}$ simplify. Indeed, instead of explicitly stating the LMIs associated with the integrality constraints $x_{i}^{2}=1, i=1, \ldots, n$, it suffices to replace (in all the other LMIs) every occurrence of a variable $y_{\alpha}$ by $y_{\beta}$ with $\beta_{i}=1$ if $\alpha_{i}>=1$, for all $i=1, \ldots, n$. This significantly reduces the number of variables in the resulting relaxation $\mathbb{Q}_{i}$.

Convergence: We make the following assumption on the set $\mathbb{K}$ defined in (6).

Assumption A: $\mathbb{K}$ is compact and there exist a polynomial $u \in \mathbb{R}\left[x_{1}, \ldots, x_{n}\right]$ of the form

$$
x \mapsto u(x):=u_{0}(x)+\sum_{i=1}^{m} u_{i}(x) g_{i}(x),
$$


for some polynomials $\left\{u_{i}\right\}$, all sums of squares, and such that $\left\{x \in \mathbb{R}^{n} \mid u(x) \geq 0\right\}$ is compact.

Assumption A is satisfied in many cases of interest. For instance, it holds as soon as $\left\{x \in \mathbb{R}^{n} \mid g_{k}(x) \geq 0\right\}$ is compact for some $k \in\{1, \ldots, m\}$, or when all the $g_{i}$ are linear and $\mathbb{K}$ is compact (hence a convex polytope), for $0-1$ (and more generally discrete) programs. In addition, if one knows that a global minimizer $x^{*}$ of $\mathbb{P}$ satisfies $\left\|x^{*}\right\| \leq M$ for some $M>0$, then adding the constraint $M-\|x\| \geq 0$ in the definition (6) of $\mathbb{K}$ will ensure that Assumption A holds.

Under Assumption A it was proved in [Las01] that inf $\mathbb{Q}_{i} \uparrow \inf \mathbb{P}$ as $i \rightarrow \infty$. Moreover, if $g_{0}(x)-p^{*}$ has the representation (7), that is,

$$
x \mapsto g_{0}(x):=q_{0}(x)+\sum_{i=1}^{m} q_{i}(x) g_{i}(x),
$$

for some polynomials $\left\{q_{i}\right\}$, all sums of squares, and of degree at most $2 i_{0}$, then for all $j \geq i_{0}$,

$$
\sup \mathbb{Q}_{j}^{*}=\max \mathbb{Q}_{j}^{*}=\min \mathbb{Q}_{j}=\inf \mathbb{Q}_{j}=\min \mathbb{P}=p^{*} .
$$

In addition, any optimal solution of $\mathbb{Q}_{j}$ identifies the vector of moments of a probability measure with mass concentrated on some global minimizers of $\mathbb{P}$.

\section{Illustration}

In this section we describe a small numerical examples to illustrate the basic use of GloptiPoly. We consider non-convex quadratic problem [Flo99, $\mathrm{Pb}$. 3.5]:

$$
\begin{array}{ll}
\min & -2 x_{1}+x_{2}-x_{3} \\
\text { s.t. } & x_{1}\left(4 x_{1}-4 x_{2}+4 x_{3}-20\right)+x_{2}\left(2 x_{2}-2 x_{3}+9\right)+x_{3}\left(2 x_{3}-13\right)+24 \geq 0 \\
& x_{1}+x_{2}+x_{3} \leq 4, \quad 3 x_{2}+x_{3} \leq 6 \\
& 0 \leq x_{1} \leq 2, \quad 0 \leq x_{2}, \quad 0 \leq x_{3} \leq 3 .
\end{array}
$$

To define this problem with GloptiPoly we use the following Matlab script:

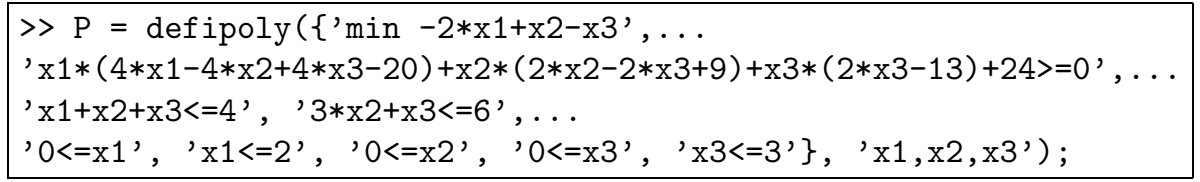

In the above script, we use features of the Symbolic Math Toolbox version 2.1, the Matlab gateway to the kernel of Maple $\mathrm{V}$ [Map01]. It is also possible to enter problems into GloptiPoly without symbolic computations, see [Glo02] for more information.

To solve the first LMI relaxation of the quadratic problem, we type:

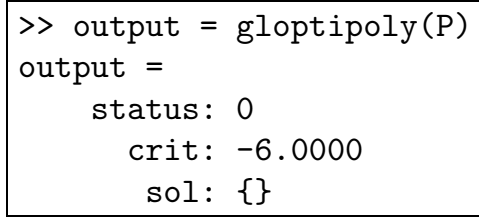


Field status $=0$ indicates that it is not possible to detect global optimality with this LMI relaxation, hence crit $=-6.0000$ is a lower bound on the global optimum.

Next we try to solve the second, third and fourth LMI relaxations of the quadratic problem with the instructions:

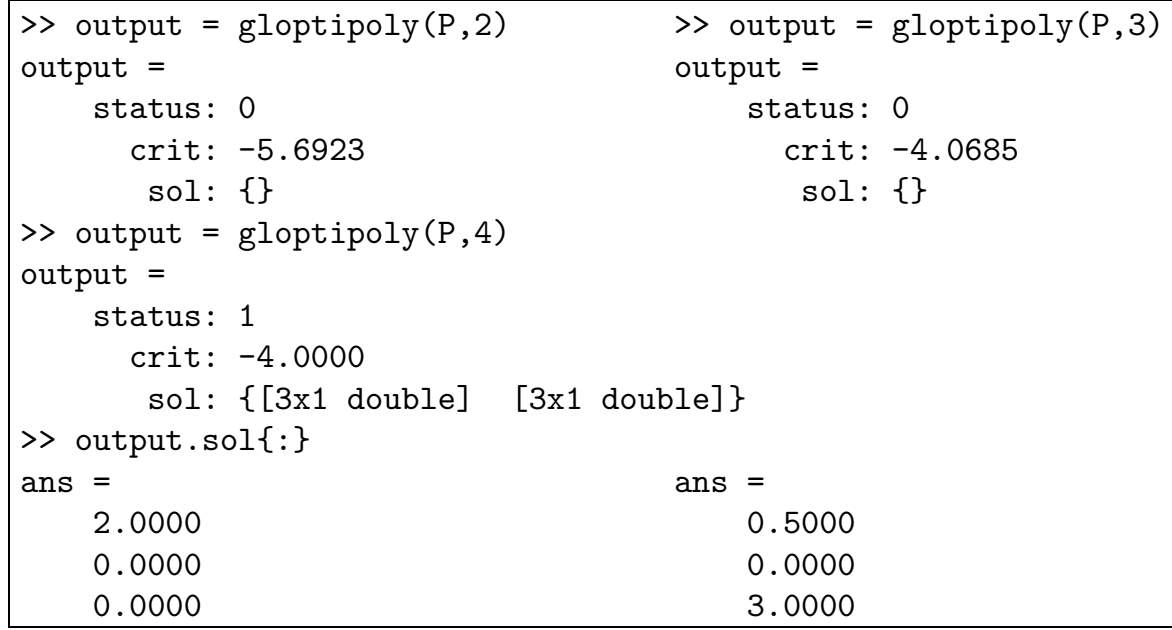

Both the second and third LMI relaxations return tighter lower bounds on the global optimum. Eventually global optimality is reached at the fourth LMI relaxation (certified by status $=1$ ). GloptiPoly also returns two globally optimal solutions $x_{1}=2, x_{2}=0, x_{3}=0$ and $x_{1}=0.5, x_{2}=0, x_{3}=3$ leading to crit $=-4.0000$.

As shown below, the number of LMI variables and the size of the relaxed LMI problem, hence the overall computational time, increase quickly with the relaxation order:

\begin{tabular}{l|c|c|c|c|c|c} 
LMI order & 1 & 2 & 3 & 4 & 5 & 6 \\
\hline LMI optimum & -6.0000 & -5.6923 & -4.0685 & -4.0000 & -4.0000 & -4.0000 \\
LMI variables & 9 & 34 & 83 & 164 & 285 & 454 \\
LMI size & 24 & 228 & 1200 & 4425 & 12936 & 32144
\end{tabular}

\section{Features}

As shown by the above numerical example, GloptiPoly is designed to solve an LMI relaxation of a given order, so it can be invoked iteratively with increasing orders until the global optimum is reached. Asymptotic convergence of the optimal values of the LMI relaxations to the global optimal value of the original problem is ensured when the compact set $\mathbb{K}$ of feasible solutions satisfies Assumption A. This condition is satisfied in many practical optimization problems, see [Las01,Las02].

General features of GloptiPoly are listed below: 
- Certificate of global optimality

- Automatic extraction of globally optimal solutions

- 0-1 or \pm 1 integer constraints on some of the decision variables (combinatorial optimization problems)

- Generation of input and output data in SeDuMi's format

- Generation of moment matrices associated with LMI relaxations

- User-defined scaling of decision variables

- Exploits sparsity of polynomial data.

Finally note that for technical reasons there is currently a limitation on the number of variables handled by GloptiPoly. For example, the current version of GloptiPoly is not able to handle quadratic problems with more than 19 variables. This limitation should be removed soon. For more details, see [Glo02].

\section{Performance}

All the computations in this section were carried out with Matlab 6.1 and SeDuMi 1.05 with relative accuracy pars.eps $=1 \mathrm{e}-9$ on a $\mathrm{PC}$ with a Pentium IV 1.6 Mhz processor with $512 \mathrm{Mb}$ RAM.

\subsection{Continuous optimization problems}

We report in Table 1 the performance of GloptiPoly on a series of benchmark non-convex continuous optimization problems. For each problem we indicated the number of decision variables 'var', the number of inequality or equality constraints 'cstr', and the maximum degree arising in the polynomial expressions 'deg'. In almost all reported instances the global optimum was reached exactly by an LMI relaxation of small order, reported in the column entitled 'order'. CPU times are in seconds. 'LMI var' is the dimension of SeDuMi dual vector $y$, whereas 'LMI size' is the dimension of SeDuMi primal vector $x$, see [SDM99]. As indicated by the label 'dim' in the rightmost column, quadratic problems 2.8, 2.9 and 2.11 in [Flo99] involve more than 19 variables and could not be handled by the current version of GloptiPoly. Except for problems 2.4 and 3.2, the computational load is moderate.

\subsection{Discrete optimization problems}

We also report the performance of GloptiPoly on a series of small-size combinatorial optimization problems. In Table 2 we first let GloptiPoly converge to the global optimum, in general extracting several solutions. The number of extracted solutions is reported in the column entitled 'sol'.

Then, we slightly perturbed the criterion to be optimized in order to destroy the problem symmetry. Proceeding this way, the optimum solution is generically unique and convergence to the global optimum is ensured more easily, cf. Table 3. See also [Glo02] for more details on this technique. 


\subsection{Polynomial systems of equations}

Multivariate polynomial systems of equations can be solved with GloptiPoly. We tested its performance on a series of benchmark examples taken from [Ver99] and [Fri00], where we removed examples featuring complex coefficients (recall that GloptiPoly handles real-valued polynomials only). Short descriptions of the benchmarks are given in Tables 4 and 5 .

We carried out our experiments by solving feasibility problems, i.e. no criterion was optimized. We did not attempt to count or enumerate all the solutions to the polynomial systems of equations, since this is outside the scope of GloptiPoly. Note that in the absence of a criterion to optimize, GloptiPoly solves the LMI relaxations by minimizing the trace of the moment matrix. Alternative criteria (such as e.g. minimum coordinate or minimum Euclidean-norm solution) are of course possible, but not investigated here.

Our results are reported in Tables 6 and 7. Column 'sol' indicates the number of solutions successfully extracted by GloptiPoly. In the last column the label 'mem' means that the error message 'out of memory' was issued by SeDuMi. GloptiPoly successfully solved about $90 \%$ of the systems.

\section{Conclusion}

GloptiPoly is as a general-purpose software with a user-friendly interface to solve in a unified way a wide range of small- to medium-size non-convex polynomial optimization problems. As illustrated by extensive numerical examples, the main strength of GloptiPoly is that no expert tuning is necessary to cope with very distinct problems coming from different branches of engineering and applied mathematics. GloptiPoly can be used as a black-box software, so it cannot be considered as a competitor to highly specialized codes for solving e.g. sparse polynomial systems of equations or large combinatorial optimization problems.

It is well-known that problems involving polynomial bases with monomials of increasing powers are naturally badly conditioned. If lower and upper bounds on the optimization variables are available as problem data, it may be a good idea to scale all the intervals around one. Alternative bases such as Chebyshev polynomials may also prove useful.

Finally, it would be instructive to compare GloptiPoly with the recently developed software SOSTOOLS [SOS02], also invoking SeDuMi to solve sums of squares optimization programs over polynomials, based on the theory described in $[$ Par00].

\section{Acknowledgment}

Work of the first author was partially supported by the Grant Agency of the Czech Republic under Project No. 102/02/0709. 


\section{References}

[Anj01] M. Anjos. New Convex Relaxations for the Maximum Cut and VLSI Layout Problems. PhD Thesis, Waterloo University, Ontario, Canada, 2001. See orion.math. uwaterloo.ca/ hwolkowi

[Flo99] C. A. Floudas, P. M. Pardalos, C. S. Adjiman, W. R. Esposito, Z. H. Gümüs, S. T. Harding, J. L. Klepeis, C. A. Meyer, C. A. Schweiger. Handbook of Test Problems in Local and Global Optimization. Kluwer Academic Publishers, Dordrecht, 1999. See titan.princeton.edu/TestProblems

[Fri00] The Numerical Algorithms Group Ltd. FRISCO - A Framefork for Integrated Symbolic/Numeric Computation. European Commission Project No. 21-024, Esprit Reactive LTR Scheme, 2000. See www.nag.co.uk/projects/frisco.html

[GW95] M. X. Goemans, D. P. Williamson. Improved approximation algorithms for maximum cut and satisfiability problems using semidefinite programming. Journal of the ACM, Vol. 42, pp. 1115-1145, 1995.

[Glo02] D. Henrion, J. B. Lasserre. GloptiPoly: Global Optimization over Polynomials with Matlab and SeDuMi. LAAS-CNRS Report No. 02057, Toulouse, France, 2002. Version 2.1 available at www.laas.fr/ henrion/software/gloptipoly

[Las01] J. B. Lasserre. Global Optimization with Polynomials and the Problem of Moments. SIAM Journal on Optimization, Vol. 11, No. 3, pp. 796-817, 2001.

[Las02] J. B. Lasserre. An Explicit Equivalent Positive Semidefinite Program for 0-1 Nonlinear Programs. SIAM Journal on Optimization, Vol. 12, No. 3, pp. 756-769, 2002.

[Map01] Waterloo Maple Software Inc. Maple V release 5. 2001. See www . maplesoft.com

[Mat01] The MathWorks Inc. Matlab version 6.1. 2001. See www.mathworks.com

[Nes00] Y. Nesterov. Squared functional systems and optimization problems. Chapter 17, pp. 405-440 in H. Frenk, K. Roos, T. Terlaky (Editors). High performance optimization. Kluwer Academic Publishers, Dordrecht, 2000.

[Par00] P. A. Parrilo. Structured Semidefinite Programs and Semialgebraic Geometry Methods in Robustness and Optimization. PhD Thesis, California Institute of Technology, Pasadena, California, 2000. See www. control.ethz.ch/ parrilo

[Put93] M. Putinar. Positive polynomials on compact semi-algebraic sets. Indiana University Mathematics Journal, Vol. 42, pp. 969-984, 1993.

[SA90] H. D. Sherali, W. P. Adams. A hierarchy of relaxations between the continuous and convex hull representations for zero-one programming problems. SIAM Journal on Discrete Mathematics, Vol. 3, pp. 411-430, 1990.

[SA99] H. D. Sherali, W. P. Adams. A reformulation-linearization technique for solving discrete and continuous nonconvex problems, Kluwer Academic Publishers, Dordrecht, 1999.

[Sho87] N. Z. Shor. Quadratic optimization problems. Tekhnicheskaya Kibernetika, Vol. 1, pp. 128-139, 1987.

[Sho98] N. Z. Shor. Nondifferentiable Optimization and Polynomial Problems. Kluwer Academic Publishers, Dordrecht, 1998.

[SDM99] J. F. Sturm. Using SeDuMi 1.02, a Matlab Toolbox for Optimization over Symmetric Cones. Optimization Methods and Software, Vol. 11-12, pp. 625-653, 1999. Version 1.05 available at fewcal.kub.nl/sturm/software/sedumi.html

[SOS02] S. Prajna, A. Papachristodoulou, P. A. Parrilo. SOSTOOLS: Sum of Squares Optimization Toolbox for Matlab. California Institute of Technology, Pasadena, USA, 2002. Version 1.00 available at www.cds.caltech.edu/sostools 
[VB96] L. Vandenberghe, S. Boyd. Semidefinite programming. SIAM Review, Vol. 38, pp. 49-95, 1996.

[Ver99] J. Verschelde. Algorithm 795: PHCpack: A general-purpose solver for polynomial systems by homotopy continuation. ACM Transactions on Mathematical Software, Vol. 25, No. 2, pp. 251-276, 1999. Database of polynomial systems available at www. math.uic.edu/ jan/demo.html

\begin{tabular}{c||c|c|c|c|c|c|c} 
problem & var & cstr & deg & LMI var & LMI size & CPU & order \\
\hline [Las01, Ex. 1] & 2 & 0 & 4 & 14 & 36 & 0.13 & 2 \\
[Las01, Ex. 2] & 2 & 0 & 4 & 14 & 36 & 0.13 & 2 \\
[Las01, Ex. 3] & 2 & 0 & 6 & 152 & 2025 & 1.13 & 8 \\
[Las01, Ex. 5] & 2 & 3 & 2 & 14 & 63 & 0.22 & 2 \\
\hline [Flo99, Pb. 2.2] & 5 & 11 & 2 & 461 & 7987 & 11.8 & 3 \\
[Flo99, Pb. 2.3] & 6 & 13 & 2 & 209 & 1421 & 1.86 & 2 \\
[Flo99, Pb. 2.4] & 13 & 35 & 2 & 2379 & 17885 & 1012 & 2 \\
[Flo99, Pb. 2.5] & 6 & 15 & 2 & 209 & 1519 & 1.58 & 2 \\
[Flo99, Pb. 2.6] & 10 & 31 & 2 & 1000 & 8107 & 67.7 & 2 \\
[Flo99, Pb. 2.7] & 10 & 25 & 2 & 1000 & 7381 & 75.3 & 2 \\
[Flo99, Pb. 2.8] & 20 & 10 & 2 & - & - & - & dim \\
[Flo99, Pb. 2.9] & 24 & 10 & 2 & - & - & - & dim \\
[Flo99, Pb. 2.10] & 10 & 11 & 2 & 1000 & 5632 & 45.3 & 2 \\
[Flo99, Pb. 2.11] & 20 & 10 & 2 & - & - & - & dim \\
\hline [Flo99, Pb. 3.2] & 8 & 22 & 2 & 3002 & 71775 & 3032 & 3 \\
[Flo99, Pb. 3.3] & 5 & 16 & 2 & 125 & 1017 & 1.20 & 2 \\
[Flo99, Pb. 3.4] & 6 & 16 & 2 & 209 & 1568 & 1.50 & 2 \\
[Flo99, Pb. 3.5] & 3 & 8 & 2 & 164 & 4425 & 2.42 & 4 \\
\hline [Flo99, Pb. 4.2] & 1 & 2 & 6 & 6 & 34 & 0.17 & 3 \\
[Flo99, Pb. 4.3] & 1 & 2 & 50 & 50 & 1926 & 0.94 & 25 \\
[Flo99, Pb. 4.4] & 1 & 2 & 5 & 6 & 34 & 0.25 & 3 \\
[Flo99, Pb. 4.5] & 1 & 2 & 4 & 4 & 17 & 0.14 & 2 \\
[Flo99, Pb. 4.6] & 2 & 2 & 6 & 27 & 172 & 0.41 & 3 \\
[Flo99, Pb. 4.7] & 1 & 2 & 6 & 6 & 34 & 0.20 & 3 \\
[Flo99, Pb. 4.8] & 1 & 2 & 4 & 4 & 17 & 0.16 & 2 \\
[Flo99, Pb. 4.9] & 2 & 5 & 4 & 14 & 73 & 0.31 & 2 \\
[Flo99, Pb. 4.10] & 2 & 6 & 4 & 44 & 697 & 0.58 & 4
\end{tabular}

Table 1. Continuous optimization problems. CPU times and LMI relaxation orders required to reach global optima. 


\begin{tabular}{c||c|c|c|c|c||c|c|c} 
problem & var & cstr & deg & LMI var & LMI size & CPU & order & sol \\
\hline QP [Flo99, Pb. 13.2.1.1] & 4 & 4 & 2 & 10 & 29 & 0.10 & 1 & 1 \\
QP [Flo99, Pb. 13.2.1.2] & 10 & 0 & 2 & 385 & 3136 & 3.61 & 2 & 1 \\
\hline Max-Cut $P_{1}$ [Flo99, Pb. 11.3] & 10 & 0 & 2 & 847 & 30976 & 38.1 & 3 & 10 \\
Max-Cut $P_{2}$ [Flo99, Pb. 11.3] & 10 & 0 & 2 & 847 & 30976 & 43.7 & 3 & 2 \\
Max-Cut $P_{3}$ [Flo99, Pb. 11.3] & 10 & 0 & 2 & 847 & 30976 & 43.0 & 3 & 2 \\
Max-Cut $P_{4}$ [Flo99, Pb. 11.3] & 10 & 0 & 2 & 847 & 30976 & 38.8 & 3 & 2 \\
Max-Cut $P_{5}$ [Flo99, Pb. 11.3] & 10 & 0 & 2 & - & - & - & 4 & dim \\
Max-Cut $P_{6}$ [Flo99, Pb. 11.3] & 10 & 0 & 2 & 847 & 30976 & 43.0 & 3 & 2 \\
Max-Cut $P_{7}$ [Flo99, Pb. 11.3] & 10 & 0 & 2 & 847 & 30976 & 44.3 & 3 & 4 \\
Max-Cut $P_{8}$ [Flo99, Pb. 11.3] & 10 & 0 & 2 & 847 & 30976 & 43.4 & 3 & 2 \\
Max-Cut $P_{9}$ [Flo99, Pb. 11.3] & 10 & 0 & 2 & 847 & 30976 & 49.3 & 3 & 6 \\
\hline Max-Cut cycle $C_{5}[$ Anj01] & 5 & 0 & 2 & 31 & 676 & 0.19 & 3 & 10 \\
Max-Cut complete $K_{5}[$ Anj01] & 5 & 0 & 2 & 31 & 961 & 0.19 & 4 & 20 \\
Max-Cut 5-node [Anj01] & 5 & 0 & 2 & 31 & 676 & 0.24 & 3 & 6 \\
Max-Cut antiweb $A W_{9}^{2}$ [Anj01] & 9 & 0 & 2 & - & - & - & 4 & dim \\
Max-Cut 10-node Petersen [Anj01] & 10 & 0 & 2 & 847 & 30976 & 39.6 & 3 & 10 \\
Max-Cut 12-node [Anj01] & 12 & 0 & 2 & - & - & - & 3 & dim
\end{tabular}

Table 2. Discrete optimization problems. CPU times and LMI relaxation orders required to reach global optima and extract several solutions.

\begin{tabular}{c||c|c|c|c|c|c|c} 
problem & var & cstr & deg & LMI var & LMI size & CPU & order \\
\hline QP [Flo99, Pb. 13.2.1.1] & 4 & 4 & 2 & 10 & 29 & 0.06 & 1 \\
QP [Flo99, Pb. 13.2.1.2] & 10 & 0 & 2 & 847 & 30976 & 40.0 & 3 \\
\hline Max-Cut $P_{1}$ [Flo99, Pb. 11.3] & 10 & 0 & 2 & 385 & 3136 & 3.10 & 2 \\
Max-Cut $P_{2}$ [Flo99, Pb. 11.3] & 10 & 0 & 2 & 385 & 3136 & 3.03 & 2 \\
Max-Cut $P_{3}$ [Flo99, Pb. 11.3] & 10 & 0 & 2 & 385 & 3136 & 3.98 & 2 \\
Max-Cut $P_{4}$ [Flo99, Pb. 11.3] & 10 & 0 & 2 & 385 & 3136 & 3.70 & 2 \\
Max-Cut $P_{5}$ [Flo99, Pb. 11.3] & 10 & 0 & 2 & 385 & 3136 & 3.41 & 2 \\
Max-Cut $P_{6}$ [Flo99, Pb. 11.3] & 10 & 0 & 2 & 385 & 3136 & 3.66 & 2 \\
Max-Cut $P_{7}$ [Flo99, Pb. 11.3] & 10 & 0 & 2 & 385 & 3136 & 3.70 & 2 \\
Max-Cut $P_{8}$ [Flo99, Pb. 11.3] & 10 & 0 & 2 & 385 & 3136 & 3.33 & 2 \\
Max-Cut $P_{9}$ [Flo99, Pb. 11.3] & 10 & 0 & 2 & 385 & 3136 & 4.03 & 2 \\
\hline Max-Cut cycle $C_{5}[$ Anj01] & 5 & 0 & 2 & 30 & 256 & 0.22 & 2 \\
Max-Cut complete $K_{5}$ [Anj01] & 5 & 0 & 2 & 31 & 676 & 0.28 & 3 \\
Max-Cut 5-node [Anj01] & 5 & 0 & 2 & 30 & 256 & 0.22 & 2 \\
Max-Cut antiweb $A W_{9}^{2}[$ Anj01] & 9 & 0 & 2 & 465 & 16900 & 12.5 & 3 \\
Max-Cut 10-node Petersen [Anj01] & 10 & 0 & 2 & 385 & 3136 & 3.14 & 2 \\
Max-Cut 12-node [Anj01] & 12 & 0 & 2 & 793 & 6241 & 29.2 & 2
\end{tabular}

Table 3. Discrete optimization problems. CPU times and LMI relaxation orders required to reach global optima with perturbed criterion. 


\begin{tabular}{|c|c|}
\hline problem & short description \\
\hline boon & neurophysiology problem \\
\hline bifur & non-linear system bifurcation \\
\hline brown & Brown's 5-dimensional almost linear system \\
\hline butcher & Butcher's system from PoSSo test suite \\
\hline camera1s & displacement of camera between two positions \\
\hline caprasse & Caprasse's system from PoSSo test suite \\
\hline cassou & Cassou-Nogues's system from PoSSo test suite \\
\hline chemequ & chemical equilibrium of hydrocarbon combustion \\
\hline cohn2 & Cohn's modular equations for special algebraic number fields \\
\hline cohn3 & Cohn's modular equations for special algebraic number fields \\
\hline comb3000 & combustion chemistry example for a temperature of 3000 degrees \\
\hline conform1 & Emiris' conformal analysis of cyclic molecules $\left(b_{11}=-9\right)$ \\
\hline conform 2 & Emiris' conformal analysis of cyclic molecules $\left(b_{11}=-\sqrt{3} / 2\right)$ \\
\hline conform3 & Emiris' conformal analysis of cyclic molecules $\left(b_{11}=-310\right)$ \\
\hline conform 4 & Emiris' conformal analysis of cyclic molecules $\left(b_{11}=-13\right)$ \\
\hline cpdm5 & 5-dimensional system of Caprasse and Demaret \\
\hline d1 & sparse system by Hong and Stahl \\
\hline des18_3 & dessin d'enfant \\
\hline $\operatorname{des} 22 \_24$ & dessin d'enfant \\
\hline discret3 & from PoSSo test suite \\
\hline eco5 & 5-dimensional economics problem \\
\hline eco6 & 6-dimensional economics problem \\
\hline eco7 & 7-dimensional economics problem \\
\hline eco8 & 8-dimensional economics problem \\
\hline fourbar & four-bar mechanical design problem \\
\hline geneig & generalized eigenvalue problem \\
\hline heart & heart dipole problem \\
\hline i1 & interval arithmetic benchmark \\
\hline ipp & six-revolute-joint problem of mechanics \\
\hline katsura5 & problem of magnetism in physics \\
\hline kinema & robot kinematics problem \\
\hline kin1 & inverse kinematics of an elbow manipulator \\
\hline ku10 & 10-dimensional system of $\mathrm{Ku}$ \\
\hline lorentz & equilibrium points of 4-dimensional Lorentz attractor \\
\hline manocha & intersection of high-degree polynomial curves \\
\hline noon3 & neural network modeled by adaptive Lotka-Volterra system \\
\hline noon 4 & neural network modeled by adaptive Lotka-Volterra system \\
\hline noon5 & neural network modeled by adaptive Lotka-Volterra system \\
\hline proddeco & system with product-decomposition structure \\
\hline puma & hand position and orientation of PUMA robot \\
\hline quadfor2 & Gaussian quadrature formula with 2 knots and 2 weights over $[-1,+1]$ \\
\hline quadgrid & interpolating quadrature formula for function defined on a grid \\
\hline
\end{tabular}

Table 4. Short descriptions of polynomial systems of equations. Part 1. 


\begin{tabular}{c|c} 
problem & short description \\
\hline rabmo & optimal multi-dimensional quadrature formulas \\
rbpl & generic positions of parallel robot \\
redeco5 & reduced 5-dimensional economics problem \\
redeco6 & reduced 6-dimensional economics problem \\
redeco7 & reduced 7-dimensional economics problem \\
redeco8 & reduced 8-dimensional economics problem \\
rediff3 & 3-dimensional reaction-diffusion problem \\
reimer5 & 5-dimensional system of Reimer \\
rose & general economic equilibrium problem \\
s9_1 & small system from constructive Galois theory \\
sendra & from PoSSo test suite \\
solotarev & from PoSSo test suite \\
stewart1 & direct kinematic problem of parallel robot \\
stewart2 & direct kinematic problem of parallel robot \\
trinks & from PoSSo test suite \\
virasoro & construction of Virasoro algebras \\
wood & system derived from optimizing the Wood function \\
wright & Wright's system
\end{tabular}

Table 5. Short descriptions of polynomial systems of equations. Part 2.

\begin{tabular}{c||c|c|c|c|c||c|c|c} 
problem & var & cstr & deg & LMI var & LMI size & CPU & order & sol \\
\hline boon & 6 & 6 & 4 & 3002 & 52864 & 1220 & 4 & 8 \\
bifur & 3 & 3 & 9 & 454 & 8717 & 8.20 & 5 & 2 \\
brown & 5 & 5 & 5 & 461 & 4061 & 6.27 & 3 & 1 \\
butcher & 7 & 7 & 4 & 6434 & 120156 & - & 4 & mem \\
camera1s & 6 & 6 & 2 & 209 & 952 & 1.33 & 2 & 2 \\
caprasse & 4 & 4 & 4 & 209 & 1285 & 0.58 & 3 & 2 \\
cassou & 4 & 4 & 8 & 4844 & 280151 & - & 8 & mem \\
chemequ & 5 & 5 & 3 & 461 & 3661 & 9.48 & 3 & 1 \\
chemequs & 5 & 5 & 3 & 124 & 486 & 6.73 & 2 & 1 \\
cohn2 & 4 & 4 & 6 & 209 & 1229 & 0.48 & 3 & 1 \\
cohn3 & 4 & 4 & 6 & 209 & 1229 & 0.55 & 3 & 1 \\
comb3000 & 10 & 10 & 3 & 1000 & 4951 & 24.6 & 2 & 1 \\
conform1 & 3 & 3 & 4 & 83 & 430 & 0.22 & 3 & 2 \\
conform2 & 3 & 3 & 4 & 83 & 430 & 0.19 & 3 & 2 \\
conform3 & 3 & 3 & 4 & 285 & 3766 & 3.89 & 5 & 4 \\
conform4 & 3 & 3 & 4 & 454 & 8946 & 12.2 & 6 & 2 \\
cpdm5 & 5 & 5 & 3 & 125 & 446 & 0.24 & 2 & 1 \\
d1 & 12 & 12 & 3 & - & - & - & 3 & dim \\
des18_3 & 8 & 8 & 3 & 12869 & 303945 & - & 4 & mem \\
des22_24 & 10 & 10 & 2 & 1000 & 5016 & 77.2 & 1 & 1 \\
discret3 & 8 & 8 & 2 & 44 & 89 & 0.31 & 1 & 1
\end{tabular}

Table 6. Polynomial systems of equations. CPU times and LMI relaxation orders required to reach global optima. Part 1. 


\begin{tabular}{|c|c|c|c|c|c|c|c|c|}
\hline problem & |var & estr & $\operatorname{leg}$ & LMI & MI size & $|\mathrm{CPU}|$ & rder & sol \\
\hline eco5 & 5 & 5 & 3 & 461 & 3661 & \begin{tabular}{|l|}
5.98 \\
\end{tabular} & 3 & 1 \\
\hline eco6 & 6 & 6 & 3 & 923 & 7980 & 57.4 & 3 & 1 \\
\hline eco7 & 7 & 7 & 3 & 1715 & 15921 & 256 & 3 & 1 \\
\hline eco8 & 8 & 8 & 3 & 3002 & 29565 & 1310 & 3 & 1 \\
\hline fourbar & 4 & 4 & 4 & 69 & 229 & 0.16 & 2 & 1 \\
\hline geneig & 6 & 6 & 3 & 923 & 7602 & 33.2 & 3 & 1 \\
\hline heart & 8 & 8 & 4 & 3002 & 31545 & 1532 & 3 & 2 \\
\hline i1 & 10 & 10 & 3 & 1000 & 4366 & 44.1 & 2 & 1 \\
\hline ipp & 8 & 8 & 2 & 494 & 2385 & 6.42 & 2 & 1 \\
\hline katsura5 & 6 & 6 & 2 & 209 & 952 & 0.74 & 2 & 1 \\
\hline kinema & 9 & 9 & 2 & 714 & 3520 & 26.4 & 2 & 1 \\
\hline kin1 & 12 & 12 & 3 & - & - & - & 3 & dim \\
\hline ku10 & 10 & 10 & 2 & 1000 & 5016 & 72.5 & 2 & 1 \\
\hline lorentz & 4 & 4 & 2 & 209 & 1705 & 0.64 & 2 & 2 \\
\hline manocha & 2 & 2 & 8 & 90 & 826 & 1.27 & 6 & 1 \\
\hline noon3 & 3 & 3 & 3 & 83 & 430 & 0.22 & 3 & 1 \\
\hline noon4 & 4 & 4 & 3 & 209 & 1285 & 0.65 & 3 & 1 \\
\hline noon5 & 5 & 5 & 3 & 461 & 3241 & 4.48 & 3 & 1 \\
\hline proddeco & 4 & 4 & 4 & 69 & 229 & 0.11 & 2 & 1 \\
\hline puma & 8 & 8 & 2 & 3002 & 35505 & 1136 & 3 & 4 \\
\hline quadfor2 & 4 & 4 & 4 & 209 & 1495 & 0.75 & 3 & 2 \\
\hline quadgrid & 5 & 5 & 5 & 461 & 3641 & 10.52 & 3 & 1 \\
\hline rabmo & 9 & 9 & 5 & 5004 & 51703 & - & 3 & mem \\
\hline $\mathrm{rbpl}$ & 6 & 6 & 3 & 923 & 7602 & 36.9 & 3 & 1 \\
\hline redeco5 & 5 & 5 & 2 & 20 & 41 & 0.16 & 1 & 1 \\
\hline redeco6 & 6 & 6 & 2 & 27 & 55 & 0.13 & 1 & 1 \\
\hline redeco7 & 7 & 7 & 2 & 35 & 71 & 0.14 & 1 & 1 \\
\hline redeco8 & 8 & 8 & 2 & 44 & 89 & 0.13 & 1 & 1 \\
\hline rediff3 & 3 & 3 & 2 & 9 & 19 & 0.09 & 1 & 1 \\
\hline reimer5 & 5 & 5 & 6 & 6187 & 264516 & - & 6 & mem \\
\hline rose & 3 & 3 & 9 & 679 & 16681 & 79.5 & 7 & 2 \\
\hline s9_1 & 8 & 8 & 2 & 494 & 2385 & 5.45 & 2 & 1 \\
\hline sendra & 2 & 2 & 7 & 65 & 453 & 0.34 & 5 & 1 \\
\hline solotarev & 4 & 4 & 3 & 69 & 257 & 0.24 & 2 & 1 \\
\hline stewart1 & 9 & 9 & 2 & 714 & 3520 & 20.4 & 2 & 2 \\
\hline stewart2 & 12 & 10 & 2 & 1819 & 9191 & 372 & 2 & 1 \\
\hline trinks & 6 & 6 & 3 & 209 & 925 & 0.78 & 2 & 1 \\
\hline virasoro & 8 & 8 & 2 & 44 & 89 & 0.16 & 1 & 1 \\
\hline wood & 4 & 3 & 2 & 69 & 527 & 0.20 & 2 & 1 \\
\hline wright & 5 & 5 & 2 & 20 & 41 & 0.17 & 1 & 1 \\
\hline
\end{tabular}

Table 7. Polynomial systems of equations. CPU times and LMI relaxation orders required to reach global optima. Part 2. 